\title{
Entropic uncertainty relations from quantum designs
}

\author{
Andreas Ketterer $\oplus^{1,2}$ and Otfried Gühne $\odot^{2}$ \\ ${ }^{1}$ Physikalisches Institut, Albert-Ludwigs-Universität Freiburg, Hermann-Herder-Str. 3, 79104 Freiburg, Germany \\ ${ }^{2}$ Naturwissenschaftlich-Technische Fakultät, Universität Siegen, Walter-Flex-Str. 3, 57068 Siegen, Germany
}

(Received 5 December 2019; accepted 26 March 2020; published 5 May 2020)

\begin{abstract}
In the course of the past decades entropic uncertainty relations have attracted much attention not only due to their fundamental role as manifestation of nonclassicality of quantum mechanics, but also as major tools for applications of quantum information theory. Amongst the latter are protocols for the detection of quantum correlations or for the secure distribution of secret keys. In this article we show how to derive entropic uncertainty relations for sets of measurements whose effects form quantum designs. The key property of quantum designs is their indistinguishability from truly random quantum processes as long as one is concerned with moments up to some finite order. Exploiting this characteristic enables us to evaluate polynomial functions of measurement probabilities which leads to lower bounds on sums of generalized entropies. As an application we use the derived uncertainty relations to investigate the incompatibility of sets of binary observables.
\end{abstract}

DOI: 10.1103/PhysRevResearch.2.023130

\section{INTRODUCTION}

Quantum mechanics prohibits observers to make simultaneous predictions about complementary properties of a physical system [1]. This fundamental aspect manifests itself through restrictions of uncertainties of measurement outcomes performed on a number of identically prepared copies of a system and is strongly related to the noncommuting structure of the observables under consideration. The latter famously lead to the formulation of Heisenberg's uncertainty relation (UR) [2] and Robertson's generalization thereof [3]. Uncertainty relations thus reflect the inherent nonclassical nature of quantum mechanics which makes them also central objects in the theory of quantum information [4,5].

The first URs have been formulated in terms of the variance of observables as a measure of their degree of uncertainty [2,3]. However, it was recognized later on that also other uncertainty measures are able to capture the concept of complementarity and, in addition, come along with a number of advantageous other properties [6]. For instance, the information theoretic interpretation of entropic uncertainty relations (EUR), which rely on entropies as quantifiers of uncertainty [6,7], makes them ideal candidates for cryptographic protocols, such as quantum key distribution or randomness generation [8]. Furthermore, EURs are an important building block for the formulation of criteria capable of detecting nonclassical properties of quantum mechanics, i.e., entanglement $[9,10]$, quantum steering [11-16], or structures of measurement incompatibility [14]. Importantly, these criteria are often applicable in discrete- as well as the continuous-variable regime, which makes them a versatile tool $[5,11,17,18]$.

Published by the American Physical Society under the terms of the Creative Commons Attribution 4.0 International license. Further distribution of this work must maintain attribution to the author(s) and the published article's title, journal citation, and DOI.
The first EURs have been derived for sums of Shannon entropies [19] and they mostly involved small sets of observables, such as position and momentum [20], or pairs of observables with finite spectrum [21]. Famously, Maassen and Uffink derived the EUR [22]:

$$
S(\mathcal{A})+S(\mathcal{B}) \geqslant \ln (1 / c), \quad c:=\max _{j, k}\left|\left\langle a_{j} \mid b_{k}\right\rangle\right|^{2},
$$

where $S(\mathcal{A})[S(\mathcal{B})]$ denotes Shannon's entropy of the probability distribution $\left\{p_{k}^{(\mathcal{A})}\right\}_{k}\left(\left\{p_{k}^{(\mathcal{B})}\right\}_{k}\right)$, originating from measurements of the observable $\mathcal{A}(\mathcal{B})$ on a quantum state $\rho$, and where the $\left|a_{j}\right\rangle$ 's $\left(\left|b_{k}\right\rangle\right)$ denote the observable's eigenstates.

Since Maassen and Uffink there have been a number of generalizations of EURs which considered generalized entropy functions as well as larger sets of observables. For instance, a number of EURs for Shannon entropies have been generalized later on to the class of Rényi [23] and Tsallis [24] entropies [22,25]. Furthermore, by invoking the properties of observables with mutually unbiased eigenbases it was possible to derive EURs involving more than two observables [26]. Also the latter have been first obtained for Shannon entropies [27,28], and were later generalized to Rényi and Tsallis entropies [29-33]. However, deriving EURs with stronger bounds or for more observables than there exist MUBs for a given dimension is a challenging task. Nevertheless, such improvements are desirable because tight EURs involving measurements in multiple bases play an important role in the security proofs of quantum cryptography applications, such as quantum key distribution [34].

In this article we take further steps in this direction and show how to derive EURs for larger sets of observables. To do so, we exploit the pseudorandom properties of the effects representing the measurements under considerations. Whenever the latter form a so-called quantum design, we are able to prove lower bounds on the sums of the respective generalized entropies. In this context, the property of being a quantum design can be seen as a natural generalization of 
the mutually unbiasedness of measurement effects, as this property can in particular be applied also to larger sets of observables than the maximum number of existing MUBs. As an application of the derived EURs we use them to study the incompatibility properties of the considered sets of binary observables.

The paper is structured as follows. In Sec. II we introduce the mathematical framework of quantum designs and present a first proposition, which is important for the subsequent discussion. We then continue in Sec. III to present our main result, a set of EURs for Rényi and Tsallis entropies involving observables whose effects form quantum designs. Examples of the derived EURs in the case of qubit systems are discussed in Sec. IV and Sec. V is devoted to one of their possible applications, i.e., the detection of the incompatibility properties of unsharp observables. Finally, we conclude in Sec. VI and give a short outlook.

\section{QUANTUM DESIGNS}

Let us denote the set of unit vectors in the finitedimensional complex Hilbert space $\mathbb{C}^{d}$ as $\mathbb{S}^{d}$. Then, a quantum $t$-design is a set $\left\{\left|\psi_{k}\right\rangle \in \mathbb{S}^{d}\right\}_{k=1}^{K}$, which fulfills the property $[35,36]$

$$
\frac{1}{K} \sum_{k=1}^{K} P_{t}\left(\left|\psi_{k}\right\rangle\right)=\int d \psi P_{t}\left(\left|\psi_{k}\right\rangle\right),
$$

where $P_{t}: \mathbb{S}^{d} \rightarrow \mathbb{C}$ denotes a polynomial of degree at most $t$ and $d \psi$ the respective Haar measure on $\mathbb{S}^{d}$. Note that every $t$-design is also a $s$-design, with $s \leqslant t$.

Quantum designs proved useful for a wide range of applications in quantum information theory [37-42]. However, though their existence has been proven [43], the known examples of exact quantum designs are rare. While there is no general strategy that allows one to generate quantum designs with a given degree $t$, one can exploit group theoretical methods to find them in a number of relevant cases $[44,45]$. In Fig. 1 we present four examples of quantum designs in $\mathbb{C}^{2}$. In some of these cases quantum designs can be associated to polyhedra with different number of vertices. For instance, the 3-design originating from the Clifford group [see Fig. 1(a)] forms an octahedron with $K_{\text {octa }}=6$ vertices, whereas the 5-designs presented in Figs. 1(b) and 1(c) correspond to an icosahedron with $K_{\text {icosa }}=12$ and an icosidodekahedron with $K_{\text {icosi }}=30$ vertices, respectively. Lastly, the 7-design with $K_{7 \text {-design }}=24$ vertices shown in Fig. 1(d) represents a deformed snub cube, i.e., a regular snub cube with slightly smaller square faces and slightly larger triangular faces [44].

An important property of quantum $t$-designs is that

$$
\sum_{k=1}^{K}\left|\psi_{k}\right\rangle\left\langle\left.\psi_{k}\right|^{\otimes t}=K \mathcal{D}_{d}^{(t)} \mathbb{1}_{t}^{\mathrm{sym}},\right.
$$

where $\mathbb{1}_{t}^{\text {sym }}$ denotes the projector onto the symmetric subspace of $\left(\mathbb{C}^{d}\right)^{\otimes t}$ and $\mathcal{D}_{d}^{(t)}=t !(d-1) ! /(t+d-1) !$ the inverse of its dimension [35]. Note that Eqs. (2) and (3) are equivalent, a fact that leads to simple methods for the verification of quantum designs [35] and to the following proposition.

Proposition 1. Given a quantum $t$-design $\left\{\left|\psi_{k}\right\rangle \in \mathbb{S}^{d}\right\}_{k=1}^{K}$ and an arbitrary density matrix $\rho \in \mathbb{C}^{d \times d}$. Then, Eq. (3)
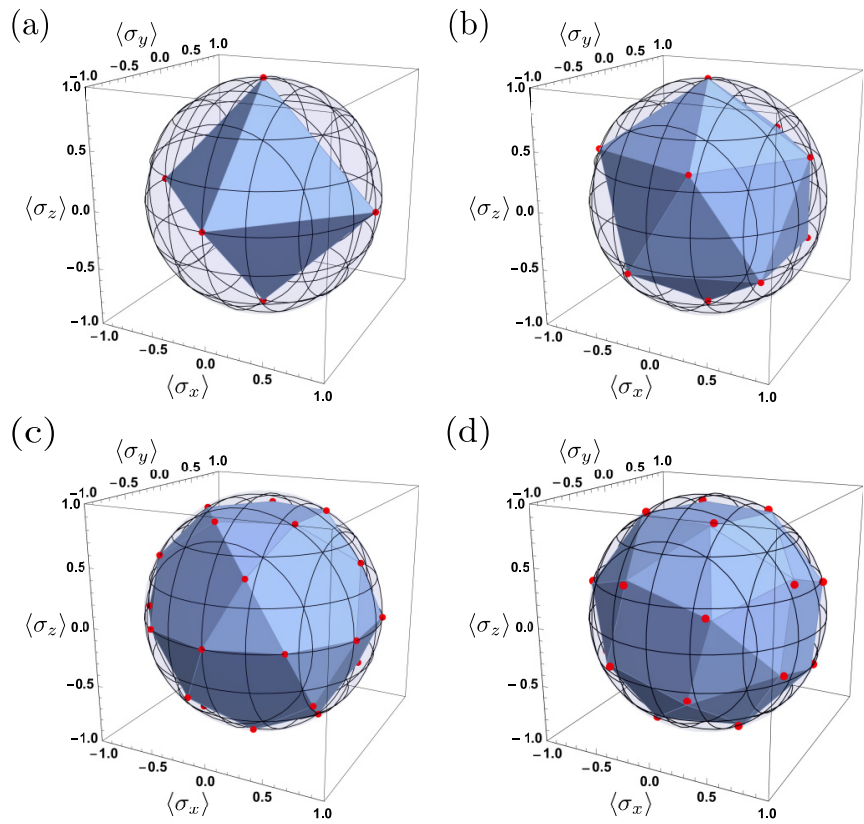

(d)

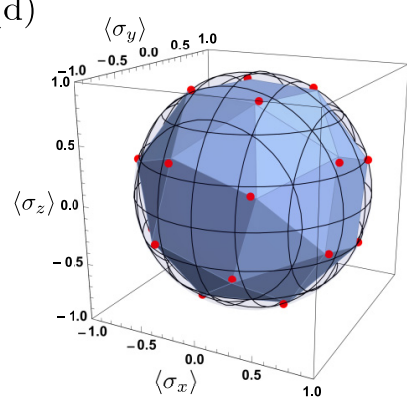

FIG. 1. Plot of the Bloch vectors of various quantum designs, for $d=2$, and their corresponding polyhedra. The components of the Bloch vector are denoted as $\left\langle\sigma_{i}\right\rangle$, with the Pauli matrices $\sigma_{x}$, $\sigma_{y}$, and $\sigma_{z}$. (a) 3-design with $K_{\text {octa }}=6$ vertices forming an octahedron. (b) 5-design with $K_{\text {icosa }}=12$ vertices forming an icosahedron. (c) 5-design with $K_{\text {icosi }}=30$ vertices forming an icosidodekahedron. (d) 7-design with $K_{7 \text {-design }}=24$ vertices forming a deformed snub cube (red points). For comparison, a regular snub cube only forms a 3-design (blue polyhedron).

implies

$$
\sum_{k=1}^{K}\left\langle\psi_{k}|\rho| \psi_{k}\right\rangle^{t}=K \mathcal{D}_{d}^{(t)} F_{t}(\rho),
$$

where $F_{t}(\rho):=\operatorname{tr}\left[\rho^{\otimes t} \mathbb{1}_{t}^{\text {sym }}\right]$.

We note that $F_{t}(\rho)$ can be expressed as a sum of monomials of the moments $\mu_{m}=\operatorname{tr}\left[\rho^{m}\right]$, with $m \leqslant t$, over the conjugacy classes of the symmetric group $S_{t}$ of permutations of $t$ elements. The latter are conveniently identified with the shape of Young diagrams or the partitions of $t$, denoted as $\lambda \vdash t$ [46], leading to

$$
F_{t}(\rho):=\frac{1}{t !} \sum_{\lambda \vdash t} h_{\lambda} \prod_{m^{(\lambda)}=1}^{t} \operatorname{tr}\left[\rho^{m^{(\lambda)}}\right]^{k_{m}^{(\lambda)}},
$$

where $h_{\lambda}$ denotes the number of permutations contained in the conjugacy class $\lambda \vdash t$, the integers $m^{(\lambda)}$ the respective orders of the cycles associated with it, and $k_{m}^{(\lambda)}$ the number of times a cycle of order $m^{(\lambda)}$ occurs. As an example, for $t=2$, we find that $F_{2}(\rho)=\left(1+\operatorname{tr}\left[\rho^{2}\right]\right) / 2$ is a linear function of the purity of $\rho$. For $t>2, F_{t}(\rho)$ involves higher moments of $\rho$ and their powers, respectively. For instance, for $t=3$ and 4 we find the two expressions

$$
\begin{gathered}
F_{3}(\rho)=\frac{1}{6}\left(1+3 \operatorname{tr}\left[\rho^{2}\right]+2 \operatorname{tr}\left[\rho^{3}\right]\right), \\
F_{4}(\rho)=\frac{1}{24}\left(1+6 \operatorname{tr}\left[\rho^{2}\right]+3 \operatorname{tr}\left[\rho^{2}\right]^{2}+8 \operatorname{tr}\left[\rho^{3}\right]+6 \operatorname{tr}\left[\rho^{4}\right]\right) .
\end{gathered}
$$



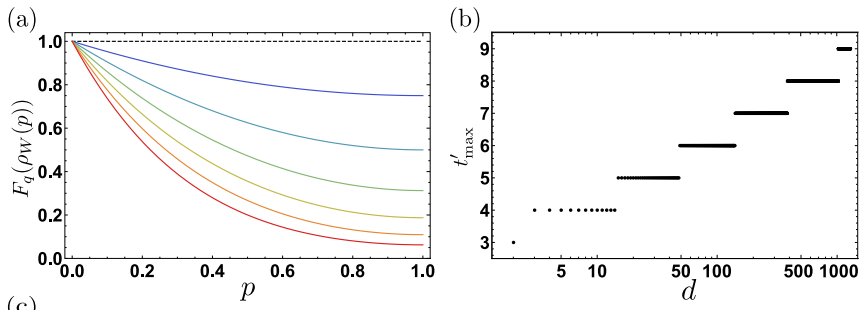

(c)

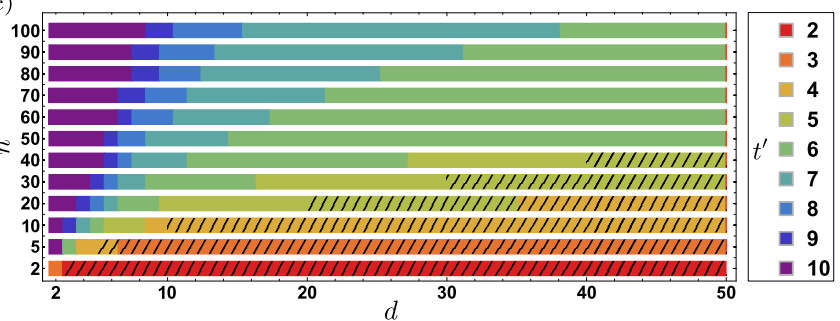

FIG. 2. (a) Plot of $F_{t}[\tilde{\rho}(p)]$ as a function of the mixing parameter $p$ for different values $t=1$ (black, dashed) and $t=2, \ldots, 7$ (blue to red). (b) Optimal value of $t^{\prime}$, resulting from the maximization of the RHS of Eq. (10) for projective measurements $(d=n)$, as a function of the dimension $d$. (c) Optimal value of $t^{\prime}$, resulting from the maximization of Eq. (10) for one rank-1 POVM $(M=1)$, as a function of the number of outcomes $n=2,5,10, \ldots, 100$ and the dimension $d=1, \ldots, 50$. The dashed area, i.e., values of $n$ and $d$ for which $n<d$, is excluded because we consider only rank-1 measurements.

For further details of the derivation of Eq. (A2) and more examples of the expression $F_{t}(\rho)$, we refer the reader to Appendix A.

Concerning the properties of Eq. (A2), it is easy to see that $F_{t}(\rho) \leqslant 1$, with equality if and only if $\rho$ is a pure state, leading directly to a state independent upper bound of the LHS of Eq. (4). Furthermore, as $\mu_{m}$ attains its minimum $1 / d^{m-1}$ for the maximally mixed state, we find that $F_{t}(\rho)$ is lower bounded by $\left[\Pi_{k=1}^{t-1}(k+d)\right] / t ! d^{t-1}$. See Fig. 2(a) for an exemplary plot of $F_{t}[\tilde{\rho}(p)]$, where $\tilde{\rho}(p):=(1-p)|0\rangle\langle 0|+$ $p \mathbb{1} / 2$, with $0 \leqslant p \leqslant 1$. It is evident that $F_{t}[\tilde{\rho}(p)]$ decreases monotonously towards the above minimum.

After having introduced the concept of quantum designs we move on and show in the next section how their properties, in particular Proposition 1, can be used to derive EURs.

\section{EURs FROM QUANTUM DESIGNS}

In the following we will focus on so-called Rényi and Tsallis entropies:

$$
\begin{aligned}
& H_{\alpha}\left(\left\{p_{k}\right\}_{k=1}^{n}\right)=\frac{1}{1-\alpha} \ln \left(\sum_{k=1}^{n} p_{k}^{\alpha}\right), \\
& T_{q}\left(\left\{p_{k}\right\}_{k=1}^{n}\right)=\frac{1}{1-q}\left(\sum_{k=1}^{n} p_{k}^{q}-1\right),
\end{aligned}
$$

with $\alpha, q \in \mathbb{R}_{>0} \backslash\{1\}$, which converge to the well-known Shannon entropy in the limit $\alpha, q \rightarrow 1$. The entropies (8) and (9) are related through the monotonic function $f_{r}(x):=$ $\ln [1+(1-r) x] /(1-r)$, with $r>1$, which allows one to convert their respective values into each other. However, we emphasize that this is in general not possible for EURs in terms of the two entropies.

As in Eq. (1), we define the entropy of a positive operator valued measure (POVM) $\mathcal{E}$ consisting of a set of effects $\left\{E_{k}\right\}_{k=1}^{n}$, with $E_{k} \geqslant 0$ and $\sum_{k} E_{k}=\mathbb{1}$, by applying Eqs. (8) or (9) to the resulting outcome probability distribution $\left\{p_{k}^{(\mathcal{E})}=\right.$ $\left.\operatorname{tr}\left[E_{k} \rho\right]\right\}_{k}$. In the particular case of a rank-1 measurement all the effects are rank-1 operators of the form $E_{k}=d / n\left|\psi_{k}\right\rangle\left\langle\psi_{k}\right|$, with a projector $\left|\psi_{k}\right\rangle\left\langle\psi_{k}\right|$ onto the one-dimensional subspace defined by $\left|\psi_{k}\right\rangle$.

With the resulting entropies as a measure for the degree of uncertainty of a measurement in hand we arrive at our main result.

Theorem 1. Given a quantum $t$-design $\left\{\left|\psi_{k}\right\rangle \in \mathbb{S}^{d}\right\}_{k=1}^{K}$, which originates from the effects of $M$ rank-1 POVMs $\left\{\mathcal{B}_{m}\right\}_{m=1}^{M}$, each having $n$ outcomes (i.e., $K=n M$ ). Then, we obtain the following EUR for Rényi entropies:

$$
\sum_{m=1}^{M} H_{\alpha}\left(\mathcal{B}_{m}\right) \geqslant \frac{M \alpha}{t^{\prime}(1-\alpha)} \ln \left(\frac{d^{t^{\prime}}}{n^{\left(t^{\prime}-1\right)}} \mathcal{D}_{d}^{\left(t^{\prime}\right)} F_{t^{\prime}}(\rho)\right),
$$

for all $\alpha \geqslant t^{\prime}$, with $t^{\prime}$ being an arbitrary integer in the interval $[2, t]$, and where $\mathcal{D}_{d}^{\left(t^{\prime}\right)}$ and $F_{t^{\prime}}(\rho)$ are defined in Proposition 1. Setting $F_{t^{\prime}}(\rho)$ equal to one yields the corresponding state independent version of Eq. (10).

Proof. Evaluating the sum of entropies in Eq. (10) yields

$$
\begin{aligned}
\sum_{m=1}^{M} H_{\alpha}\left(\mathcal{B}_{m}\right) & =\frac{1}{1-\alpha} \sum_{k=1}^{M} \ln \left(\sum_{k=1}^{n} \operatorname{tr}\left[\rho \frac{d}{n}\left|b_{k}^{(m)}\right\rangle\left\langle b_{k}^{(m)}\right|\right]^{\alpha}\right) \\
& =\frac{1}{1-\alpha} \sum_{k=1}^{M} \ln \left[\sum_{k=1}^{n}\left(\frac{d}{n}\right)^{\alpha}\left\langle b_{k}^{(m)}|\rho| b_{k}^{(m)}\right\rangle^{\alpha}\right],
\end{aligned}
$$

where $\left\{\left|b_{k}^{(m)}\right\rangle\right\}_{k}$ denotes the set of eigenstates corresponding to the effects of the $m$ th POVM $\mathcal{B}_{m}=\left\{n / d\left|b_{k}^{(m)}\right\rangle\left\langle b_{k}^{(m)}\right|\right\}_{k=1}^{n}$. Next, we use the monotonicity of the $p$-norm $|\boldsymbol{x}|_{p}$, which states that

$$
|\boldsymbol{x}|_{q}=\left(\sum_{j} x_{j}^{q}\right)^{1 / q} \leqslant\left(\sum_{j} x_{j}^{p}\right)^{1 / p}=|\boldsymbol{x}|_{p},
$$

for $1 \leqslant p<q \leqslant \infty$, and apply it to the argument of the logarithm in Eq. (12). This, in combination with the fact that the function $f_{\alpha}(x):=\ln (x) /(1-\alpha)$ is monotonically decreasing for $\alpha>1$, allows us to estimate the RHS of Eq. (12) and arrive at

$$
\begin{aligned}
\sum_{m=1}^{M} H_{\alpha}\left(\mathcal{B}_{m}\right) & \geqslant \frac{1}{1-\alpha} \sum_{k=1}^{M} \ln \left\{\left[\sum_{k=1}^{n}\left(\frac{d}{n}\right)^{t^{\prime}}\left\langle b_{k}^{(m)}|\rho| b_{k}^{(m)}\right\rangle^{t^{\prime}}\right]^{\frac{\alpha}{t^{\prime}}}\right\} \\
& =\frac{\alpha}{t^{\prime}(1-\alpha)} \sum_{k=1}^{M} \ln \left[\sum_{k=1}^{n}\left(\frac{d}{n}\right)^{t^{\prime}}\left\langle b_{k}^{(m)}|\rho| b_{k}^{(m)}\right\rangle^{t^{\prime}}\right] .
\end{aligned}
$$


Further on, we use the convexity of $f_{\alpha}(x)$, for $\alpha \geqslant 1$, and Jensen's inequality to get

$$
\begin{aligned}
& \frac{\alpha}{t^{\prime}(1-\alpha)} \sum_{k=1}^{M} \ln \left[\sum_{k=1}^{n}\left(\frac{d}{n}\right)^{t^{\prime}}\left\langle b_{k}^{(m)}|\rho| b_{k}^{(m)}\right\rangle^{t^{\prime}}\right] \\
& \geqslant \frac{\alpha M}{t^{\prime}(1-\alpha)} \ln \left(\frac{d^{t^{\prime}}}{M n^{t^{\prime}}} \sum_{k=1}^{M} \sum_{k=1}^{n}\left\langle b_{k}^{(m)}|\rho| b_{k}^{(m)}\right\rangle^{t^{\prime}}\right) .
\end{aligned}
$$

Now, as the union of all eigenstates $\left\{\left|b_{k}^{(m)}\right\rangle\right\}_{k, m}$ corresponding to the POVMs $\left\{\mathcal{B}_{m}\right\}_{m=1}^{M}$ forms a quantum $t$-design, we can apply Proposition 1 according to which we find

$$
\sum_{m=1}^{M} \sum_{k=1}^{n}\left\langle b_{k}^{(m)}|\rho| b_{k}^{(m)}\right\rangle^{t^{\prime}}=n M \mathcal{D}_{d}^{\left(t^{\prime}\right)} F_{t^{\prime}}(\rho) \leqslant n M \mathcal{D}_{d}^{\left(t^{\prime}\right)},
$$

for all $t^{\prime} \leqslant t$, and apply it to Eq. (16), which finally yields

$$
\sum_{m=1}^{M} H_{\alpha}\left(\mathcal{B}_{m}\right) \geqslant \frac{M \alpha}{t^{\prime}(1-\alpha)} \ln \left(\frac{d^{t^{\prime}}}{n^{\left(t^{\prime}-1\right)}} \mathcal{D}_{d}^{\left(t^{\prime}\right)} F_{t^{\prime}}(\rho)\right),
$$

for all $\alpha \geqslant t^{\prime}$, with an integer $1<t^{\prime} \leqslant t$. Lastly, imposing $F_{t^{\prime}}(\rho) \leqslant 1$, for all $\rho$, yields the corresponding state independent form of Eq. (18):

$$
\sum_{m=1}^{M} H_{\alpha}\left(\mathcal{B}_{m}\right) \geqslant \frac{M \alpha}{t^{\prime}(1-\alpha)} \ln \left(\frac{d^{t^{\prime}}}{n^{\left(t^{\prime}-1\right)}} \mathcal{D}_{d}^{\left(t^{\prime}\right)}\right) .
$$

Note that an analog version of Theorem 1 involving Tsallis entropies is presented in Appendix C. Also, due to the generality of Theorem 1, Eq. (10) yields in particular EURs for sets of projective measurements $(n=d)$, and bounds on the entropy of single rank-1 POVMs with $n$ elements $(M=1)$ [47].

However, before we discuss concrete examples, we note that Theorem 1 provides us with a whole set of EURs parametrized by the value of the parameter $t^{\prime}$ [see Eq. (10)]. The latter arises due to the fact that a given set of observables whose effects form a quantum $t$-design also form a $t^{\prime}$-design, with $0<t^{\prime} \leqslant t$. Hence we have to maximize the lower bound in Eq. (10) in order to obtain the optimal EUR for a given set of measurements. We discuss this maximization for the two above mentioned cases of projective measurements $(d=n)$, and individual POVMs $(M=1)$ [48]. The result is presented in Figs. 2(b) and 2(c), respectively. For projective measurements we find that, while for $d=2$ the best bound is obtained for $t^{\prime}=3$, for an increasing dimension larger values of $t^{\prime}$ yield better EUR bounds. In the second case, i.e., for individual POVMs, we find that large values of $t^{\prime}$ become useful for increasing the number of outcomes $n$ depending on the dimension $d$. Also, we emphasize that the bounds of the state dependent version of Eq. (10) become stronger for increasingly mixed states, as the minimum of $F_{t}(\rho)$ is attained for the maximally mixed one [see also Fig. 2(a)].

Lastly, we want to stress that the existence of the EURs derived in Theorem 1 is conditioned on the existence of a set of observables having the desired design property. This is reminiscent of EURs for measurements in mutually unbiased bases (MUB) [28,30-32], which can be proven for any dimension while complete sets of $d+1$ MUBs are not known
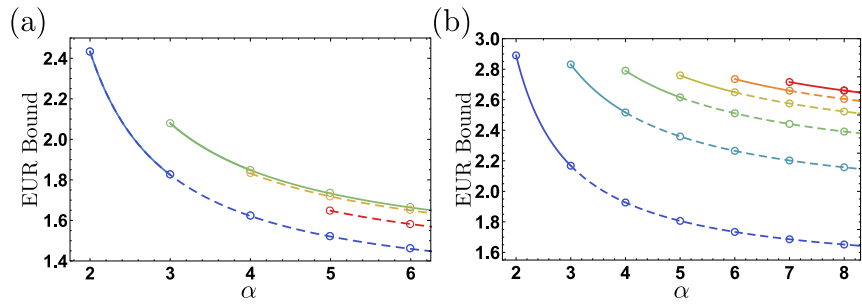

FIG. 3. (a) Plot of the bound from Eq. (21) as a function of the parameter $\alpha$ for different values $t^{\prime}=2$ (blue), 3 (green), 4 (yellow), and 5 (red). (b) Plot of the bound on the Rényi entropy of the individual POVM $\mathcal{E}^{\text {(7-design) }}$, consisting of the 7-design presented in Fig. 1(d), as a function of the parameter $q$ for different values $t^{\prime}=2$ (blue), 3 (cyan), 4 (green), 5 (yellow), 6 (orange), and 7 (red). Solid lines represent the strongest bounds within a given range of $\alpha$ and $q$ and the circles indicate integer values.

for arbitrary $d$ [41]. Note that Eq. (10) implies the bounds obtained for full sets of MUBs, as the latter were proven by exploiting the fact that MUBs form quantum 2-designs [30]. Hence our procedure generalizes the bounds obtained for MUBs to larger sets of observables, as will be discussed in terms of several examples in Sec. IV.

\section{EXAMPLES FOR QUBIT SYSTEMS}

As concrete examples of the introduced EURs we consider sets of qubit observables whose eigenstates $\left|v_{i}^{(m)}\right\rangle$, with $i=0,1$, form one of the quantum designs presented in Fig. 1. In the case of the octahedron [Fig. 1(a)], Theorem 1 leads to the well-known EURs for the three MUBs, $\mathcal{X}=\{|+\rangle\langle+||-\rangle,\langle-|\}, \mathcal{Y}=\left\{\left|+{ }_{i}\right\rangle\left\langle+_{i}||-,{ }_{i}\right\rangle\left\langle-{ }_{i}\right|\right\}$, and $\mathcal{Z}=$ $\{|0\rangle\langle 0|| 1\rangle,\langle 1|\}[30,31]$. In particular, since qubit MUBs form not only 2-designs but also 3-designs, we improve the Rényi version of these EURs for $\alpha \geqslant 3$. In this case, we find

$$
\begin{aligned}
& H_{\alpha}(\mathcal{X})+H_{\alpha}(\mathcal{Y})+H_{\alpha}(\mathcal{Z}) \\
& \quad \geqslant\left\{\begin{array}{lll}
\frac{3 \alpha}{2(1-\alpha)} \ln \left(\frac{2}{3}\right), & \text { for } \quad 2 \leqslant \alpha<3, \\
\frac{\alpha}{(1-\alpha)} \ln \left(\frac{1}{2}\right), & \text { for } \quad \alpha \geqslant 3 .
\end{array}\right.
\end{aligned}
$$

Further on, for the icosahedron 5-design, presented in Fig. 1(b), Theorem 1 yields the following EUR for a set of six qubit measurements $\left\{\mathcal{B}_{m}^{\text {(icosa) }}\right\}_{m=1}^{6}$ :

$$
\sum_{m=1}^{6} H_{\alpha}\left(\mathcal{B}_{m}^{\text {(icosa) }}\right) \geqslant \frac{6 \alpha}{t^{\prime}(1-\alpha)} \ln \left(\frac{2 t^{\prime} !}{\left(1+t^{\prime}\right) !}\right),
$$

for all $\alpha, q \geqslant t^{\prime}$, with an integer $1<t^{\prime} \leqslant 5$.

Equation (21), together with its Tsallis counterpart, presented in Appendix B, thus constitute two concrete examples of EURs involving a larger number of observables than the maximal number of MUBs for $d=2$. We present their bounds as functions of $\alpha$ in Fig. 3(a) [see Appendix B for a discussion of the Tsallis version of Eq. (21)]. We see that the optimal bound of Eq. (21) is reached for $t^{\prime}=2$ in the range $2 \leqslant \alpha \leqslant 3$ and for $t^{\prime}=3$ when $\alpha \geqslant 3$, leading to

$$
\sum_{m=1}^{6} H_{\alpha}\left(\mathcal{B}_{m}^{\text {(icosa) })}\right) \geqslant \begin{cases}\frac{3 \alpha}{1-\alpha} \ln (2 / 3), & \text { for } \quad 2 \leqslant \alpha<3 \\ \frac{2 \alpha}{1-\alpha} \ln (1 / 2), & \text { for } \quad \alpha \geqslant 3\end{cases}
$$


We emphasize that the EUR (21) cannot be obtained by trivially combining EURs for two sets of measurements of MUB, as the condition $\left|\left\langle v_{i}^{\left(m_{1}\right)} \mid v_{j}^{\left(m_{2}\right)}\right\rangle\right|=1 / \sqrt{2}$ is not fulfilled for any combination of $i, j, m_{1}$, and $m_{2}$. This situation changes for a measurement constructed from the icosidodekahedron 5-design [see Fig. 1(c)], which can be equally obtained by combining a set of five rotated MUBs.

Lastly, we note that the 7-design presented in Fig. 1(d) does not consist of pairs of mutually orthogonal states preventing us from constructing a set of projective qubits measurements from it. However, as discussed previously, Theorem 1 also provides bounds on the entropy of individual POVMs. For instance, in the case of the POVM $\mathcal{E}^{(7 \text {-design })}=\left\{24 / 2\left|\kappa_{i}\right\rangle\left\langle\kappa_{i}\right|\right\}_{i=1}^{24}$ made of the 24 element improved snub cube 7-design, presented in Fig. 1(d), we find

$$
H_{\alpha}\left(\mathcal{E}^{(7-\text { design })}\right) \geqslant \frac{\alpha}{(\alpha-1) t^{\prime}} \ln \left(\frac{t^{\prime}+1}{2^{3-2 t^{\prime}} \times 3^{1-t^{\prime}}}\right),
$$

for $\alpha \geqslant t^{\prime}$ and with $t^{\prime} \leqslant 7$. Figure 3(b) shows a plot of the bounds (23) for different values of $t^{\prime}$. As expected from Fig. 2(c), increasing values of $t^{\prime}$ yield better bounds for growing $\alpha$. However, as $t^{\prime} \leqslant 7$ one cannot reach the optimal value of $t^{\prime}=10$.

\section{DETECTION OF MEASUREMENT INCOMPATIBILITY}

As an application of the above derived EURs we use them to investigate the incompatibility properties of the previously discussed measurements. To do so, we adopt the notion of joint measurability which defines a set of measurements $\left\{\mathcal{E}_{m}\right\}_{m=1}^{M}$ to be compatible if its effects $E_{k \mid m}$ can be obtained via a classical postprocessing of a larger joint measurement $\mathcal{G}=\left\{G_{\lambda}\right\}_{\lambda}$, i.e., $E_{k \mid m}=\sum_{\lambda} p(k \mid m, \lambda) G_{\lambda}$. If for a given set of measurements such a joint measurement does not exist we call them incompatible or not jointly measurable.

In order to investigate the incompatibility of a given set of measurements $\left\{\mathcal{A}_{m}\right\}_{m=1}^{M}$ we make use of the equivalence between steering and joint measurability problems [49,50]. More precisely, if two parties, Alice and Bob, share a pure maximally entangled state, the ability of Alice to steer Bob's state is equivalent to her using measurements which are not jointly measurable. Hence we can exploit entropic steering inequalities (ESI) in order to test whether Alice's measurements are jointly measurable.

There have been several works devoted to the derivation of quantum steering inequalities from entropic uncertainty relations [11-14]. These derivations were first based on EURs involving Shannon entropies [11,12], but have been generalized to Tsallis and Rényi entropies later on [13,14]. In the following, we focus on the approach presented in Ref. [14] as it is the only one applicable to EURs involving Rényi entropies for arbitrary $\alpha$.

The main result of Ref. [14] is a one-to-one mapping between any state independent EURs of the form

$$
\sum_{m} H_{m}\left(\mathcal{A}_{m}\right) \geqslant q\left(\left\{\mathcal{A}_{m}\right\}\right)
$$

with entropies $H_{m}$ and state independent bound $q\left(\left\{\mathcal{A}_{m}\right\}\right)$, and entropic steering inequalities (ESI) of the form

$$
\sum_{m=1}^{M} H_{m}\left(\mathcal{B}_{m} \mid \mathcal{A}_{m}\right) \geqslant q\left(\left\{\mathcal{B}_{m}\right\}_{m=1}^{M}\right)
$$

where $H_{m}\left(\mathcal{B}_{m} \mid \mathcal{A}_{m}\right)$ denotes the respective conditional entropy [see Eq. (28) for a definition], provided the following two conditions hold.

(i) The considered EUR holds true when conditioned on any classical random variable $\mathcal{Y}$,

$$
\sum_{m} H_{m}\left(\mathcal{A}_{m} \mid \mathcal{Y}\right) \geqslant q\left(\left\{\mathcal{A}_{m}\right\}\right)
$$

(ii) The considered entropies are nonincreasing under conditioning on additional information, i.e.,

$$
H_{m}\left(\mathcal{X} \mid \mathcal{Y}_{1}\right) \geqslant H_{m}\left(\mathcal{X} \mid \mathcal{Y}_{1}, \mathcal{Y}_{2}\right)
$$

Here, we make use of this formalism to derive ESIs from EURs for Rényi entropies $H_{\alpha}$, with $\alpha \geqslant 2$. The associated conditional Rényi entropies are defined as follows:

$$
H_{\alpha}\left(\mathcal{P}_{1} \mid \mathcal{P}_{2}\right)=\frac{\alpha}{1-\alpha} \ln \left(\sum_{y} p_{y}^{(2)}\left|\boldsymbol{p}_{x \mid y}^{(1 \mid 2)}\right|_{\alpha}\right),
$$

where $|\boldsymbol{x}|_{\alpha}$ denotes the $\alpha$-norm and $\boldsymbol{p}_{\boldsymbol{x} \mid y}^{(1 \mid 2)}$ is a vector containing conditional probabilities $p_{x \mid y}^{(1 \mid 2)}$ for a fixed $y$. Conditions (i) and (ii) have been proven in Refs. [14] and [51], respectively. In this respect, the authors of Ref. [14] focused on EURs involving at most two entropies; however, a generalization of the presented argument to more measurements is straightforward. All in all, this leads us to the following ESI presented in Eq. (9) of the main text:

$$
\sum_{m=1}^{M} H_{\alpha}\left(\mathcal{B}_{m} \mid \mathcal{A}_{m}\right) \geqslant q\left(\left\{\mathcal{B}_{m}\right\}_{m=1}^{M}\right)
$$

where $H_{\alpha}(X \mid Y)$ denotes the conditional Rényi entropy as defined in Eq. (28) and $q\left(\left\{\mathcal{B}_{m}\right\}_{m=1}^{M}\right)$ denotes the bound of the corresponding state independent Rényi EUR for Bob's measurements $\left\{\mathcal{B}_{m}\right\}_{m=1}^{M}$.

To test this procedure we regard the corresponding noisy counterparts $\mathcal{A}_{m}^{\eta}$ of Alice's measurements $\mathcal{A}_{m}=\left\{A_{k \mid m}\right\}$, defined as $A_{k \mid m}^{\eta}=\eta A_{k \mid m}+(1-\eta) \mathbb{1} / d$, with $0 \leqslant \eta \leqslant 1$, and determine for which threshold value of $\eta \mathrm{Eq}$. (25) is violated. Starting with the case of three MUBs, i.e., $\mathcal{A}^{\text {(Pauli) }}=$ $\{\mathcal{X}, \mathcal{Y}, \mathcal{Z}\}$, we recover that for $\alpha=2$ and 3 a violation of Eq. (25) occurs for the well-known threshold $\eta \geqslant 1 / \sqrt{3}$ showing their triplewise incompatibility [52]. Further on, we are able to apply the same procedure to the previously introduced set of six icosahedron measurements $\left\{\mathcal{B}_{m}^{\text {(icosa) }}\right\}_{m=1}^{6}$, which violate the ESI for $\eta \geqslant 1 / \sqrt{3}$, with $\alpha=2$ and 3 . Hence, in terms of the robustness against noise, the measurements $\left\{\mathcal{B}_{m}^{\text {(icosa) }}\right\}_{m=1}^{6}$ are at least as incompatible as the set of Pauli measurements $\mathcal{A}=\{\mathcal{X}, \mathcal{Y}, \mathcal{Z}\}$ even though they are not mutually unbiased. 


\section{CONCLUSIONS}

We have proven bounds of entropic uncertainty relations in terms of Rényi and Tsallis entropies for measurements whose effects form quantum $t$-designs. To do so, we exploited the fact that quantum $t$-designs form projectors onto the symmetric subspace of a $t$-fold tensor product space which allowed us to make use of elements of representation theory of the symmetric group. The optimality of the introduced EURs was discussed for varying values of $t$ showing that larger degree designs can lead to stronger EUR bounds. Further on, we presented specific examples of these EURs, e.g., for measurements in mutually unbiased bases but also for larger sets of observables or for individual POVMs. Lastly, we probed the incompatibility properties of the introduced measurements through a set of steering inequalities which is based on the introduced EURs.

As an outlook one could imagine to further generalize the introduced EUR for measurements whose effects form incomplete quantum designs, as it is possible to find EURs for less than $d+1$ MUBs in dimension $d$. Another interesting prospect is to derive EURs from approximate quantum designs, i.e., designs that fulfill the condition (2) up to some small error. Such a generalization would be interesting as there exist efficient constructions of approximate designs even for large values of $t[53,54]$.

\section{ACKNOWLEDGMENTS}

We are indebted to R. Schwonnek, T. Simnacher, N. Wyderka, and X.-D. Yu for valuable discussions about entropic uncertainty relations. We acknowledge financial support from the ERC (Consolidator Grant No. 683107/TempoQ) and the DFG. A.K. acknowledges support from the Georg H. Endress foundation.

\section{APPENDIX A: PROOF OF PROPOSITION 1}

Here, we prove Proposition 1 which states that, given a quantum $t$-design $\left\{\left|\psi_{k}\right\rangle \in \mathbb{S}^{d}\right\}_{k=1}^{K}$ and an arbitrary density matrix $\rho \in \mathbb{C}^{d \times d}$, the following holds:

$$
\sum_{k=1}^{K}\left\langle\psi_{k}|\rho| \psi_{k}\right\rangle^{t}=K \mathcal{D}_{d}^{(t)} F_{t}(\rho)
$$

where $F_{t}(\rho):=\operatorname{tr}\left[\rho^{\otimes t} \mathbb{1}_{t}^{\text {sym }}\right]$, which can be expressed as a sum of monomials of the moments $\mu_{m}=\operatorname{tr}\left[\rho^{m}\right]$, with $m \leqslant t$, over the conjugacy classes of the symmetric group $S_{t}$ of permutations of $t$ elements. The latter are conveniently identified with the shape of Young diagrams or the partitions of $t$, denoted as $\lambda \vdash t$ [46], leading to

$$
F_{t}(\rho):=\frac{1}{t !} \sum_{\lambda \vdash t} h_{\lambda} \prod_{m^{(\lambda)}=1}^{t} \operatorname{tr}\left[\rho^{m^{(\lambda)}}\right]^{k_{m}^{(\lambda)}},
$$

where $h_{\lambda}$ denotes the number of permutations contained in the conjugacy class $\lambda \vdash t$, the integers $m^{(\lambda)}$ the respective orders of the cycles associated with it, and $k_{m}^{(\lambda)}$ the number of times a cycle of order $m^{(\lambda)}$ occurs.
Proof. To infer Eq. (A1) we first note that any quantum $t$-design has the following property:

$$
\sum_{k=1}^{K}\left|\psi_{k}\right\rangle\left\langle\left.\psi_{k}\right|^{\otimes t}=K \mathcal{D}_{d}^{(t)} \mathbb{1}_{t}^{\mathrm{sym}},\right.
$$

where $\mathbb{1}_{t}^{\text {sym }}$ denotes the projector on the symmetric subspace $\operatorname{Sym}^{t}\left(\mathbb{C}^{d}\right)$ of $\left(\mathbb{C}^{d}\right)^{\otimes t}$. By definition $\mathbb{1}_{t}^{\text {sym }}$ can be expressed as a sum over permutation matrices $R_{\pi}$, which are defined as follows:

$$
R_{\pi}\left|\psi_{1}\right\rangle \otimes \ldots \otimes\left|\psi_{t}\right\rangle=\left|\psi_{\pi^{-1}(1)}\right\rangle \otimes \cdots \otimes\left|\psi_{\pi^{-1}(t)}\right\rangle,
$$

where $\pi$ denotes an element of the symmetric group $S_{t}$ which contains $t$ ! elements, leading to the well-known expression

$$
\mathbb{1}_{t}^{\mathrm{sym}}=\frac{1}{t !} \sum_{\pi \in S_{t}} R_{\pi} .
$$

Now, by simply taking the expectation value of Eq. (A3) with respect to a state $\rho^{\otimes t}$ we get

$$
\sum_{k=1}^{K}\left\langle\psi_{k}|\rho| \psi_{k}\right\rangle^{t}=K \mathcal{D}_{d}^{(t)} \frac{1}{t !} \sum_{\pi \in S_{t}} \operatorname{tr}\left[\rho^{\otimes t} R_{\pi}\right] .
$$

Hence it remains to be shown that $\sum_{\pi \in S_{t}} \operatorname{tr}\left[\rho^{\otimes t} R_{\pi}\right]=$ $\sum_{\lambda \vdash t} h_{\lambda} \prod_{m=1}^{t} \operatorname{tr}\left[\rho^{m}\right]^{k_{m}^{(\lambda)}}$.

To do so, we have to review some basics concerning the representation theory of the symmetric group $S_{t}$ of permutations of $t$ elements. First, note that $S_{t}$ contains $t$ ! permutations each of which can be uniquely decomposed into a product of disjoint cyclic permutations (cycles) [55], also referred to as cycle decomposition. A cycle is of order $m$ if it acts on $m \leqslant t$ elements and the set of orders $\left\{m_{1} m_{2} \ldots\right\}$ of the cycles contained in a cycle decomposition, sorted in nonincreasing order, determine the cycle type of a permutation. Alternatively, one can denote the cycle type of a permutation $\pi$ as $\left(1^{k_{1}} 2^{k_{2}} \ldots t^{k_{t}}\right)$, where $k_{r}$ is the number a cycle of order $r$ occurs.

To make this clear we consider the following example. The permutation $\tilde{\pi}:\{12345678\} \rightarrow\{31254687\}$ of $S_{8}$ consists of the cycles $6 \rightarrow 6,4 \rightarrow 5 \rightarrow 4,7 \rightarrow 8 \rightarrow 7$, and $2 \rightarrow$ $1 \rightarrow 3 \rightarrow 2$, of order $1,2,2$, and 3 , respectively. The cycle decomposition of $\tilde{\pi}$ thus consists of $k_{1}=1$ cycles of order $1, k_{2}=2$ cycles of order 2 , and $k_{3}=1$ cycles of order 3 . The cycle type thus reads (1223), or in the above introduced notation $\left(1^{1} 2^{2} 3^{1}\right)$, where cycles which occur $k_{i}=0$ times are not incorporated.

It is evident that the cycle types of the permutations $\pi \in S_{t}$ can be identified with the partitions of the integer $t$. A partition is a sequence of nonnegative integer numbers sorted in nonincreasing order: $\lambda=\left(\lambda_{1}, \lambda_{2}, \ldots, \lambda_{r}, \ldots, \lambda_{l(\lambda)}\right)$, of length $l(\lambda)$. Specifically, a partition of an integer number $t$ is denoted by $\lambda \vdash t$, i.e., $t=\sum_{i} \lambda_{i}$, and the total number of partitions of an integer $t$ by $P_{t}$. Furthermore, the cycle types also label the conjugacy classes of $S_{t}$, i.e., the equivalence classes of permutations that are conjugate to each other. Two permutations $\pi, \pi^{\prime} \in S_{t}$ are conjugates if there exists a third permutation $\sigma \in S_{t}$, such that $\pi=\sigma \pi^{\prime} \sigma^{-1}$. The number $h_{\lambda}$ of permutations contained in the conjugacy class $\lambda$ is then determined by the respective cycle type $\left(1^{k_{1}} 2^{k_{2}} \ldots t^{k_{t}}\right)$ through 
the formula [55]

$$
h_{\lambda}=\frac{t !}{k_{1} ! 1^{k_{1}} k_{2} ! 2^{k_{2}} \times \cdots \times k_{t} ! t^{k_{t}}} .
$$

As a consequence of the above discussion, we find that each permutation matrix $R_{\pi}$, corresponding to the permutation $\pi$, can be split into a product of cyclic permutation matrices $R_{m_{1}} \otimes R_{m_{2}} \otimes \cdots \otimes R_{m_{r}}$, where the $m_{i}$ 's denote the orders of the involved cycles and $r$ the total number of cycles contained in the cycle decomposition of $\pi$. Note that each $R_{m_{i}}$ acts on a subspace $\left(\mathbb{C}^{d}\right)^{\otimes m_{i}}$ of $\left(\mathbb{C}^{d}\right)^{\otimes t}$, such that $\bigotimes_{i=1}^{r}\left(\mathbb{C}^{d}\right)^{\otimes m_{i}}=$ $\left(\mathbb{C}^{d}\right)^{\otimes t}$. If we take now the expectation value of $R_{\pi}$, where $\pi$ is a permutation of the conjugacy class $\lambda$ with cycle type $\left(1^{k_{1}^{(\lambda)}} 2^{k_{2}^{(\lambda)}} \ldots t^{k_{t}^{(\lambda)}}\right)$, with respect to the $t$-fold tensor product state $\rho^{\otimes t}$, we arrive at

$$
\begin{aligned}
\operatorname{tr}\left[\rho^{\otimes t} R_{\pi}\right] & =\operatorname{tr}\left[\rho^{\otimes m_{1}} R_{m_{1}}\right] \operatorname{tr}\left[\rho^{\otimes m_{2}} R_{m_{2}}\right] \ldots \operatorname{tr}\left[\rho^{\otimes m_{r}} R_{m_{r}}\right] \\
& =\operatorname{tr}\left[\rho R_{1}\right]^{k_{1}} \operatorname{tr}\left[\rho^{\otimes 2} R_{2}\right]^{k_{2}} \ldots \operatorname{tr}\left[\rho^{\otimes t} R_{t}\right]^{k_{t}} \\
& =\operatorname{tr}\left[\rho^{2}\right]^{k_{2}} \operatorname{tr}\left[\rho^{3}\right]^{k_{3}} \ldots \operatorname{tr}\left[\rho^{t}\right]^{k_{t}} .
\end{aligned}
$$

In the second line (A9) we grouped together terms of the same order $m_{i}$, and in line (A10) we used that the expectation value of any cyclic permutation of order $m$ with respect to a state $\rho^{\otimes m}$ is equal to $\operatorname{tr}\left[\rho^{m}\right]$, a fact that can be easily inferred from the definition (A4) of the permutation matrices $R_{\pi}$. Lastly, summing over all permutations $\pi \in S_{t}$ yields

$$
\begin{aligned}
\sum_{\pi \in S_{t}} \operatorname{tr}\left[\rho^{\otimes t} R_{\pi}\right] & =\sum_{\pi \in S_{t}} \operatorname{tr}\left[\rho^{2}\right]^{(\lambda)} \ldots \operatorname{tr}\left[\rho^{t}\right]^{k_{t}^{(\lambda)}} \\
& =\sum_{\lambda \vdash t} h_{\lambda} \operatorname{tr}\left[\rho^{2}\right]^{k_{2}^{(\lambda)}} \ldots \operatorname{tr}\left[\rho^{t}\right]^{k_{t}^{(\lambda)}} \\
& =\sum_{\lambda \vdash t} h_{\lambda} \prod_{m=1}^{t} \operatorname{tr}\left[\rho^{m}\right]^{k_{m}^{(\lambda)}},
\end{aligned}
$$

where we used that the conjugacy classes of $S_{t}$ are labeled by the partitions $\lambda$ of $t$, and that each conjugacy class contains $h_{\lambda}$ elements [according to Eq. (A7)]. This completes the proof of Proposition 1.

In order to get familiar with the structure of the function $F_{t}(\rho)$ we discuss in the following some exemplary cases. Trivially, we find that for $t=1$ we have $F_{1}(\rho)=1$, as $S_{1}$ contains only a single element. In contrast, $S_{2}$ contains exactly two elements, i.e., the identity $i d$ and the transposition $\pi_{12}$, which are cycles of order 1 and 2 , respectively. We thus find that

$$
F_{2}(\rho)=\frac{1}{2}\left(1+\operatorname{tr}\left[\rho^{2}\right]\right) .
$$

For $t=3$ the situation gets slightly more complicated as $S_{3}$ has six elements. However, it is easy to see that those elements can be grouped into three conjugacy classes according to the three possible arrangements of Young diagrams, which can be labeled by the partitions (111), (12), and (3) of $t=3$. According to Eq. (A7) we thus have that $h_{(111)}=1, h_{(12)}=$ 3 , and $h_{(3)}=2$. Using the previously introduced alternative notation of the partitions $\lambda$ as cycle types we can write $\left(1^{3}\right)$,
(12), and (3), thus inferring that

$$
F_{3}(\rho)=\frac{1}{6}\left(1+3 \operatorname{tr}\left[\rho^{2}\right]+2 \operatorname{tr}\left[\rho^{3}\right]\right) .
$$

Following the same strategy for $t=4,5,6,7$ yields

$$
\begin{aligned}
F_{4}(\rho)= & \frac{1}{24}\left(1+6 \operatorname{tr}\left[\rho^{2}\right]+3 \operatorname{tr}\left[\rho^{2}\right]^{2}+8 \operatorname{tr}\left[\rho^{3}\right]+6 \operatorname{tr}\left[\rho^{4}\right]\right), \\
F_{5}(\rho)= & \frac{1}{120}\left(1+15 \operatorname{tr}\left[\rho^{2}\right]^{2}+20 \operatorname{tr}\left[\rho^{3}\right] \operatorname{tr}\left[\rho^{2}\right]+10 \operatorname{tr}\left[\rho^{2}\right]\right. \\
& \left.+20 \operatorname{tr}\left[\rho^{3}\right]+30 \operatorname{tr}\left[\rho^{4}\right]+24 \operatorname{tr}\left[\rho^{5}\right]\right), \\
F_{6}(\rho)= & \frac{1}{720}\left(1+15 \operatorname{tr}\left[\rho^{2}\right]^{3}+45 \operatorname{tr}\left[\rho^{2}\right]^{2}+120 \operatorname{tr}\left[\rho^{3}\right] \operatorname{tr}\left[\rho^{2}\right]\right. \\
& +t 90 \operatorname{tr}\left[\rho^{4}\right] \operatorname{tr}\left[\rho^{2}\right]+15 \operatorname{tr}\left[\rho^{2}\right]+40 \operatorname{tr}\left[\rho^{3}\right]^{2} \\
& \left.+40 \operatorname{tr}\left[\rho^{3}\right]+90 \operatorname{tr}\left[\rho^{4}\right]+144 \operatorname{tr}\left[\rho^{5}\right]+120 \operatorname{tr}\left[\rho^{6}\right]\right),
\end{aligned}
$$

$$
\begin{aligned}
F_{7}(\rho)= & \frac{1}{5040}\left(1+21 \operatorname{tr}\left[\rho^{2}\right]+105 \operatorname{tr}\left[\rho^{2}\right]^{2}+105 \operatorname{tr}\left[\rho^{2}\right]^{3}\right. \\
& +70 \operatorname{tr}\left[\rho^{3}\right]+420 \operatorname{tr}\left[\rho^{2}\right] \operatorname{tr}\left[\rho^{3}\right]+210 \operatorname{tr}\left[\rho^{2}\right]^{2} \operatorname{tr}\left[\rho^{3}\right] \\
& +280 \operatorname{tr}\left[\rho^{3}\right]^{2}+210 \operatorname{tr}\left[\rho^{4}\right]+630 \operatorname{tr}\left[\rho^{2}\right] \operatorname{tr}\left[\rho^{4}\right] \\
& +420 \operatorname{tr}\left[\rho^{3}\right] \operatorname{tr}\left[\rho^{4}\right]+504 \operatorname{tr}\left[\rho^{5}\right]+504 \operatorname{tr}\left[\rho^{2}\right] \operatorname{tr}\left[\rho^{5}\right] \\
& \left.+840 \operatorname{tr}\left[\rho^{6}\right]+720 \operatorname{tr}\left[\rho^{7}\right]\right) .
\end{aligned}
$$

Lastly, we note that in Ref. [56] a similar formula has been derived in a different context using the properties of unitary designs. Unitary designs are the analogs of quantum designs on the level of unitary transformations, i.e., a set $\left\{U_{k} \in\right.$ $\mathcal{U}(d) \mid k=1, \ldots, K\}$ of unitary $(d \times d)$ matrices is called a unitary $t$-design if the following property holds:

$$
\frac{1}{K} \sum_{k=1}^{K} P_{t, t}(U)=\int d U P_{t, t}\left(U_{k}\right),
$$

where $P_{t, t}: \mathcal{U}(d) \rightarrow \mathbb{C}$ denotes a polynomial of degree at most $t$ in the entries of the respective unitary matrix and their complex conjugates and $d U$ is the Haar measure on $\mathcal{U}(d)$.

\section{APPENDIX B: THEOREM FOR TSALLIS ENTROPIES}

We can formulate an equivalent version of Theorem 1 for Tsallis entropies.

Theorem 2. Given a quantum $t$-design $\left\{\left|\psi_{k}\right\rangle \in \mathbb{S}^{d}\right\}_{k=1}^{K}$ which originates from the effects of $M$ rank-1 POVMs $\left\{\mathcal{B}_{m}\right\}_{m=1}^{M}$, each having $n$ outcomes (i.e., $K=n M$ ). Then, we obtain the following EUR for Rényi entropies:

$$
\sum_{m=1}^{M} T_{q}\left(\mathcal{B}_{m}\right) \geqslant \frac{1}{1-q}\left(\left[d M D_{d}^{\left(t^{\prime}\right)} F_{t^{\prime}}(\rho)\right]^{q / t^{\prime}}-M\right),
$$

for all $q \geqslant t^{\prime}$, with $t^{\prime}$ being an arbitrary integer in the interval $[2, t]$, and where $\mathcal{D}_{d}^{\left(t^{\prime}\right)}$ and $F_{t^{\prime}}(\rho)$ are defined as in Proposition 1. Setting $F_{t^{\prime}}(\rho)$ equal to one yields the corresponding state independent version of Eq. (B1). 
To prove Theorem 2 we first evaluate the sym of entropies in Eq. (B1), yielding

$$
\begin{gathered}
\sum_{m=1}^{M} T_{q}\left(\mathcal{B}_{m}\right)=\frac{1}{1-q} \sum_{k=1}^{M}\left(\sum_{k=1}^{n} \operatorname{tr}\left[\rho \frac{d}{n}\left|b_{k}^{(m)}\right\rangle\left\langle b_{k}^{(m)}\right|\right]^{q}-1\right) \\
=\frac{1}{q-1}\left[M-\sum_{k=1}^{M} \sum_{k=1}^{n}\left(\frac{d}{n}\right)^{\alpha}\left\langle\left. b_{k}^{(m)}|\rho| b_{k}^{(m)}\right|^{q}\right] .\right.
\end{gathered}
$$

Next, we apply the monotonicity of the $p$-norm $|\boldsymbol{x}|_{p}$ to Eq. (B2) to arrive at

$$
\begin{aligned}
& \sum_{m=1}^{M} T_{q}\left(\mathcal{B}_{m}\right) \\
& \quad \geqslant \frac{1}{q-1}\left\{M-\left(\frac{d}{n}\right)^{q}\left[\sum_{k=1}^{M} \sum_{k=1}^{n}\left\langle b_{k}^{(m)}|\rho| b_{k}^{(m)}\right\rangle^{t^{t^{\prime}}}\right]^{\frac{q}{t^{\prime}}}\right\} .
\end{aligned}
$$

Now, we use again Proposition 1 and the fact that $x^{q / t^{\prime}}$ is monotonically increasing with $x$, which yields

$$
\sum_{m=1}^{M} T_{q}\left(\mathcal{B}_{m}\right) \geqslant \frac{1}{1-q}\left(\left[\frac{d^{t^{\prime}}}{n^{t^{\prime}-1}} M \mathcal{D}_{d}^{\left(t^{\prime}\right)} F_{t^{\prime}}(\rho)\right]^{q / t^{\prime}}-M\right),
$$

for all $q \geqslant t^{\prime}$, with an integer $1<t^{\prime} \leqslant t$. Imposing $F_{t^{\prime}}(\rho) \leqslant 1$, for all $\rho$, again yields the state independent version

$$
\sum_{m=1}^{M} T_{q}\left(\mathcal{B}_{m}\right) \geqslant \frac{1}{1-q}\left(\left[\frac{d^{t^{\prime}}}{n^{t^{\prime}-1}} M \mathcal{D}_{d}^{\left(t^{\prime}\right)}\right]^{q / t^{\prime}}-M\right) .
$$

In particular, for $d=2$ with the MUBs $\mathcal{X}=$ $\{|+\rangle\langle+||-\rangle,\langle-|\}, \quad \mathcal{Y}=\left\{\left|+{ }_{i}\right\rangle\left\langle+{ }_{i}||-,{ }_{i}\right\rangle\left\langle-{ }_{i}\right|\right\}, \quad$ and $\quad \mathcal{Z}=$ $\{|0\rangle\langle 0|| 1\rangle,\langle 1|\}$, we obtain

$$
\begin{aligned}
& T_{q}(\mathcal{X})+T_{q}(\mathcal{Y})+T_{q}(\mathcal{Z}) \\
& \quad \geqslant\left\{\begin{array}{lll}
\frac{1}{1-q}\left(2^{q / 2}-3\right), & \text { for } \quad 2 \leqslant q<3, \\
\frac{1}{1-q}\left[(3 / 2)^{q / 3}-3\right], & \text { for } \quad q \geqslant 3 .
\end{array}\right.
\end{aligned}
$$
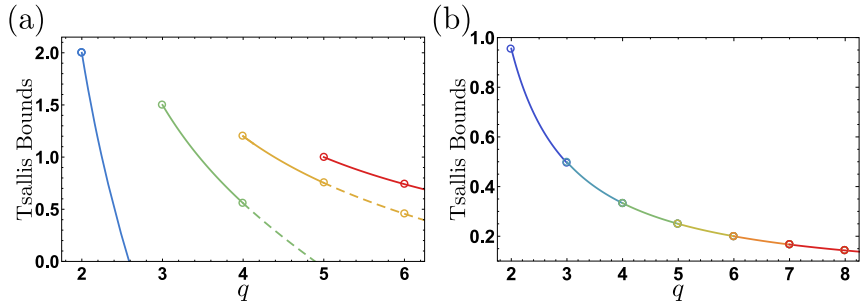

FIG. 4. (a) Plot of the bound of the EUR (B7) as a function of the parameter $\alpha$ for different values $t^{\prime}=2$ (blue), 3 (green), 4 (yellow), and 5 (red). (b) Plot of the bound on the Tsallis entropy of the individual POVM $\mathcal{E}^{(7-\text { design })}$, consisting of the 7-design presented in Fig. 1(d) of the main text, as a function of the parameter $q$ for different values $t^{\prime}=2$ (blue), 3 (cyan), 4 (green), 5 (yellow), 6 (orange), and 7 (red). Solid lines indicate for which ranges of $\alpha$ and $q$ the respective bounds are strongest and the circles emphasize integer values.

Next, we discuss the EUR for the qubit measurements $\left\{\mathcal{B}_{m}^{\text {(icosa) }}\right\}_{m=1}^{6}$ whose effects form an icosahedron:

$$
\sum_{m=1}^{6} T_{q}\left(\mathcal{I}_{m}\right) \geqslant \frac{1}{1-q}\left(\left[\frac{12 t^{\prime} !}{\left(t^{\prime}+1\right) !}\right]^{q / t^{\prime}}-6\right),
$$

for which the optimal values of $t^{\prime}$ are presented in Fig. 4(a) of the main text.

Furthermore, we also discuss bounds on entropies of individual POVMs whose effects form quantum designs. For instance, in the case of the POVM $\mathcal{E}^{(7-\text { design })}=\left\{24 / 2\left|\kappa_{i}\right\rangle\left\langle\kappa_{i}\right|\right\}_{i=1}^{24}$ made of the 24 element improved snub cube 7-design, presented in Fig. 1(d) of the main text, we find

$$
T_{q}\left(\mathcal{E}^{(7-\text { design })}\right) \geqslant \frac{1}{1-q}\left[2^{q / t^{\prime}}\left(\frac{2^{3-2 t^{\prime}} \times 3^{1-t^{\prime}}}{t^{\prime}+1}\right)^{q / t^{\prime}}-1\right],
$$

for $q \geqslant t^{\prime}$ and with $t^{\prime} \leqslant 7$. In Fig. 4(b) we present the resulting bounds of Eq. (B8) as a function of $q$ for different values of $t^{\prime}$. In the latter case varying $t^{\prime}$ has very little influence on the value of the bound. Hence $t^{\prime}=2$ can be assumed for all values of $q$.
[1] A. Messiah, Quantum Mechanics (Dover Publications Inc., Mineola, NY, 2014).

[2] W. Heisenberg, Z. Phys. 43, 172 (1927).

[3] H. Robertson, Phys. Rev. 34, 163 (1929).

[4] M. D. Reid, P. D. Drummond, W. P. Bowen, E. G. Cavalcanti, P. K. Lam, H. A. Bachor, U. L. Andersen, and G. Leuchs, Rev. Mod. Phys. 81, 1727 (2009).

[5] F. Toscano, D. S. Tasca, Ł. Rudnicki, and S. P. Walborn, Entropy 20, 454 (2018).

[6] P. J. Coles, M. Berta, M. Tomamichel, and S. Wehner, Rev. Mod. Phys. 89, 015002 (2017).

[7] S. Wehner and A. Winter, New J. Phys. 12, 025009 (2010).

[8] N. Gisin, G. Ribordy, W. Tittel, and H. Zbinden, Rev. Mod. Phys. 74, 145 (2002).

[9] O. Gühne and M. Lewenstein, Phys. Rev. A 70, 022316 (2004).
[10] O. Gühne and G. Tóth, Phys. Rep. 474, 1 (2009).

[11] S. P. Walborn, A. Salles, R. M. Gomes, F. Toscano, and P. H. Souto Ribeiro, Phys. Rev. Lett. 106, 130402 (2011).

[12] J. Schneeloch, C. J. Broadbent, S. P. Walborn, E. G. Cavalcanti, and J. C. Howell, Phys. Rev. A 87, 062103 (2013).

[13] A. C. S. Costa, R. Uola, and O. Gühne, Phys. Rev. A 98 050104(R) (2018).

[14] T. Kriváchy, F. Fröwis, and N. Brunner, Phys. Rev. A 98, 062111 (2018).

[15] D. Cavalcanti and P. Skrzypczyk, Rep. Prog. Phys. 80, 024001 (2017).

[16] R. Uola, A. C. S. Costa, H. C. Nguyen, and O. Gühne, Rev. Mod. Phys. 92, 015001 (2020).

[17] L.-M. Duan, G. Giedke, J. I. Cirac, and P. Zoller, Phys. Rev. Lett. 84, 2722 (2000). 
[18] R. Simon, Phys. Rev. Lett. 84, 2726 (2000).

[19] C. Shannon, Bell Syst. Tech. J. 27, 379 (1948).

[20] I. Białynicki-Birula and J. Mycielski, Commun. Math. Phys. 44, 129 (1975).

[21] D. Deutsch, Phys. Rev. Lett. 50, 631 (1983).

[22] H. Maassen and J. B. M. Uffink, Phys. Rev. Lett. 60, 1103 (1988).

[23] A. Rényi, in Proceedings of the 4th Berkeley Symposium on Mathematical Statistics and Probability (University of California Press, Berkeley, CA, 1961), Vol. 1, pp. 547.

[24] C. Tsallis, J. Stat. Phys. 52, 479 (1988).

[25] S. Zozor, G. M. Bosyk, and M. Portesi, J. Phys. A 47, 495302 (2014).

[26] T. Durt, B.-G. Englert, I. Bengtsson, and K. Życzkowski, Int. J. Quantum Inf. 08, 535 (2010).

[27] J. Sanchez-Ruiz, Phys. Lett. A 173, 233 (1993).

[28] S. Wu, S. Yu, and K. Molmer, Phys. Rev. A 79, 022104 (2009).

[29] M. A. Ballester and S. Wehner, Phys. Rev. A 75, 022319 (2007).

[30] A. E. Rastegin, Eur. Phys. J. D 67, 269 (2013).

[31] A. E. Rastegin, Quantum Inf. Process. 12, 2947 (2013).

[32] A. E. Rastegin, Open Syst. Inf. Dyn. 22, 1550005 (2015).

[33] J. Kaniewski, M. Tomamichel, and S. Wehner, Phys. Rev. A 90, 012332 (2014).

[34] I. B. Damgard, S. Fehr, R. Renner, L. Salvail, and C. Schaffner, in Advances in Cryptology-CRYPTO 2007, Lecture Notes in Computer Science, edited by A. Menezes, Vol. 4622 (Springer, Berlin, Heidelberg, 2007), pp. 360-378.

[35] J. M. Renes, R. Blume-Kohout, A. J. Scott, and C. M. Caves, J. Math. Phys. 45, 2171 (2004).

[36] A. Ambainis and J. Emerson, Twenty-Second Annual IEEE Conference on Computational Complexity (CCC'07) (IEEE, New York, 2007), p. 129.

[37] A. Scott, J. Phys. A 41, 055308 (2008).

[38] A. Roy and A. J. Scott, Des. Codes Cryptogr. 53, 13 (2009).
[39] C. Dankert, R. Cleve, J. Emerson, and E. Livine, Phys. Rev. A 80, 012304 (2009).

[40] D. Gross, F. Krahmer, and R. Kueng, J. Fourier Anal. Appl. 21, 229 (2015).

[41] J. Bae, B. C. Hiesmayr, and D. McNulty, New J. Phys. 21, 013012 (2019).

[42] A. Ketterer, N. Wyderka, and O. Gühne, Phys. Rev. Lett. 122, 120505 (2019).

[43] P. D. Seymour and T. Zaslavsky, Adv. Math. 52, 213 (1984).

[44] R. H. Hardin and N. J. A. Sloane, Discr. Comput. Geom. 15, 429 (1996).

[45] D. Gross, K. Audenaert, and J. Eisert, J. Math. Phys. 48, 052104 (2007).

[46] P. Flajolet and R. Sedgewick, Analytic Combinatorics (Cambridge University Press, Cambridge, UK, 2009).

[47] M. Krishna and K. R. Parthasarathy, Sankhyā: Indian J. Stat. 64, 842 (2002), arXiv:quant-ph/0110025.

[48] Note that the prefactor $\alpha /(\alpha-1)$ is monotonously decreasing, for $\alpha>1$, and its maximum is thus always obtained for $\alpha=t^{\prime}$.

[49] R. Uola, T. Moroder, and O. Gühne, Phys. Rev. Lett. 113, 160403 (2014).

[50] R. Uola, C. Budroni, O. Gühne, and J.-P. Pellonpää, Phys. Rev. Lett. 115, 230402 (2015).

[51] M. Iwamoto and J. Shikata, in Information Theoretic Security, edited by C. Padró (Springer, Cham, 2014), pp. 103-121.

[52] R. Kunjwal, C. Heunen, and T. Fritz, Phys. Rev. A 89, 052126 (2014).

[53] F. G. S. L. Brandão, A. W. Harrow, and M. Horodecki, Phys. Rev. Lett. 116, 170502 (2016).

[54] Y. Nakata, C. Hirche, M. Koashi, and A. Winter, Phys. Rev. X 7, 021006 (2017).

[55] H. Boerner, Representations of Groups (North-Holland, Amsterdam, 1970).

[56] B. Vermersch, A. Elben, M. Dalmonte, J. I. Cirac, and P. Zoller, Phys. Rev. A 97, 023604 (2018). 